\section{Ciclosporina versus tacrolimus no transplante renal no Brasil: uma comparação de custos}

\author{
Cyclosporine versus tacrolimus in kidney \\ transplants in Brazil: a cost comparison
}

\author{
Augusto Afonso Guerra Junior 1,2 \\ Francisco de Assis Acúrcio 1 \\ Eli Iola Gurgel Andrade 3 \\ Mariângela Leal Cherchiglia 3 \\ Cibele Comini Cesar 4 \\ Odilon Vanni de Queiroz 1,5 \\ Grazielle Dias da Silva ${ }^{2}$
}

\footnotetext{
${ }_{1}^{1}$ Faculdade de Farmácia, Universidade Federal de Minas Gerais, Belo Horizonte, Brasil.

2 Superintendência de Assistência Farmacêutica, Secretaria de Estado da Saúde de Minas Gerais, Belo Horizonte, Brasil.

3 Faculdade de Medicina. Universidade Federal de Minas Gerais, Belo Horizonte, Brasil.

4 Instituto de Ciências Exatas, Universidade Federal de Minas Gerais, Belo Horizonte, Brasil.

${ }^{5}$ Agência Nacional de Saúde Suplementar, Belo Horizonte, Brasil.

Correspondência A. A. Guerra Junior Departamento de Farmácia Social, Faculdade de Farmácia, Universidade Federal de Minas Gerais. Av. Presidente Antônio Carlos 6627, Belo Horizonte, $M G$ 31270-901, Brasil. augustoguerra@ig.com.br
}

\begin{abstract}
In Brazil, the Unified National Health System (SUS) is responsible for the majority of kidney transplants. To maintain these interventions, the guidelines recommend the use of cyclosporine or tacrolimus, associated with corticosteroids and azathioprine or mycophenolate. Taking the perspective of the National Health System, an economic analysis was performed on the outpatient and hospital resources and medicines used by patient and therapeutic group. A cohort was constructed from 2000 to 2004, with 5,174 kidney transplant patients in use of cyclosporine or tacrolimus, identified by probabilistic record linkage from the National Health System. The cohort in cluded 4,015 patients in use of cyclosporine and 1,159 using tacrolimus. The majority were males, age $\leq 38$ years, with nephritis, cardiovascular diseases, and indeterminate causes as the most frequent primary diagnoses. After 48 months of follow-up, the expenditures were higher for kidney transplants in hospitals in the Northeast, cadaver donors, patients in dialysis $>24$ months before the transplant, and in the tacrolimus group. Total hospital and outpatient costs and expenditure on medication were higher in patients on tacrolimus as compared to the cyclosporine group.
\end{abstract}

Kidney Transplantation; Health Expenditures; Health Economics

\section{Introdução}

O processo de transição demográfica no Brasil caracteriza-se por um rápido envelhecimento da população, o qual contribui para o crescimento das doenças crônico-degenerativas. Dentre essas, destaca-se a insuficiência renal crônica, por seu alto impacto econômico para o sistema de saúde e perda da qualidade de vida. A insuficiência renal crônica é responsável por um crescente número de pacientes submetidos às terapias de substituição renal, sejam diálises, sejam transplantes renais 1,2. Em 2007, o Sistema Único de Saúde (SUS) pagou cerca de R 2 bilhões em diálises e procedimentos relacionados, transplantes e medicamentos (http://www.datasus. gov.br, acessado em 21/Mar/2008).

O Brasil possui um dos maiores programas públicos de transplantes de órgãos e tecidos do mundo, e os transplantes renais começaram a ser realizados no país a partir da década de 1960. Em consonância com as necessidades de saúde, foi aprovada, em 2004, a Política Nacional de Atenção ao Portador de Doença Renal, na qual se direcionam ações específicas em favor do transplante 1 . Entretanto, apesar dos esforços que vêm sendo envidados, em dezembro de 2006, havia cerca de 32 mil pacientes na lista de espera para realizar transplante renal 2 .

Nesse cenário, é crescente o debate sobre modelos de atenção adotados, com vistas ao 
financiamento necessário à organização dos serviços e ao provimento dos cuidados. A literatura tem demonstrado ser o transplante a alternativa mais custo-efetiva dentre as terapias de substituição renal, pois permite, na maioria dos casos, a reintegração do paciente às suas atividades cotidianas, aumentando a probabilidade de uma melhor expectativa e qualidade de vida em relação aos pacientes submetidos às diálises 3 .

Um fator que tem contribuído, substancialmente, para que o transplante renal seja considerado a melhor opção para o portador de insuficiência renal crônica é o avanço tecnológico no que diz respeito à terapia de imunossupressão. $\mathrm{O}$ principal objetivo dessa terapia é aumentar a sobrevida do enxerto, evitando as rejeições agudas e crônicas do órgão transplantado 3,4,5.

No Brasil, desde a promulgação da Constituição Federal de 1988, o direito à saúde é universal, incluída a assistência terapêutica e farmacêutica integral. Em 2002, foram estabelecidos protocolos clínicos e diretrizes terapêuticas para uso dos medicamentos excepcionais e de alto custo no SUS 4 , dentre os quais aqueles utilizados no transplante renal ${ }^{6}$. Os protocolos clínicos recomendam, para a manutenção no transplante renal, a adoção do esquema de ciclosporina + azatioprina + corticosteróides. Alternativamente, permite-se a substituição da ciclosporina por tacrolimus. Outra possibilidade é substituir a azatioprina por micofenolato mofetil ou sirolimus.

Todavia, sabe-se da pressão pela disponibilização de recursos em virtude das muitas necessidades competitivas não atendidas, principalmente no campo da assistência farmacêutica. Apesar do impacto econômico no orçamento do SUS, ainda não se conhecem os custos diretos advindos da adoção dos diferentes esquemas imunossupressores no país, bem como da incorporação de novas tecnologias ao longo do período em estudo. Ao mesmo tempo, o SUS possui um conjunto de registros informatizados que permite conhecer, desde o momento do transplante, as principais despesas com essas terapêuticas: o Sistema de Informações Hospitalares (SIH) e ambulatorial (APAC/SIA) (http:// www.datasus.gov.br). Essas bases, em conjunto com o Sistema de Informações sobre Mortalidade (SIM) e outros dados relacionados de forma probabilística 7, conformaram a Base de Dados Nacional em terapias de substituição renal (http://www.datasus.gov.br)/ (http://www.bpre co.saude.gov.br/bprefd/owa/consulta.inicio) 8,9 .

Diante do exposto, o objetivo deste trabalho é realizar uma análise dos gastos entre pacientes transplantados no SUS, inseridos nessa base, que utilizaram ciclosporina ou tacrolimus em esquemas de manutenção imunossupressora.
Serão levantadas despesas, na perspectiva do SUS, com procedimentos hospitalares a partir do transplante renal e procedimentos ambulatoriais relacionados à inserção desses pacientes no Programa de Medicamentos de Dispensação Excepcional.

\section{Métodos}

\section{Desenho do estudo}

Coorte histórica composta por todos os pacientes que realizaram transplante renal doador vivo ou cadavérico no Brasil, em todos os centros transplantadores do SUS, no período de janeiro de 2000 a dezembro de 2003, e que fizeram uso dos medicamentos imunossupressores ciclosporina ou tacrolimus. Foram excluídos do estudo indivíduos que não apresentaram um período mínimo de três meses de uso dos esquemas imunossupressores adotados e de seis meses de sobrevida, a partir da data do transplante.

Os participantes foram identificados nas bases nacionais de dados hospitalares (SIH), ambulatoriais (APAC/SIA) e de mortalidade (SIM) do SUS, por meio de relacionamento probabilístico dos registros administrativos 7,8,9.

Este estudo é parte integrante dos projetos Avaliação Econômica e Epidemiológica das Terapias de Substituição Renal no Brasil e Avaliação Farmacoeconômica e Farmacoepidemiológica dos Medicamentos de Alto Custo no Brasil, realizados pelos Grupos de Pesquisa em Economia da Saúde e em Farmacoepidemiologia da Universidade Federal de Minas Gerais (UFMG), que foram aprovados pelo Comitê de Ética em Pesquisa da UFMG (pareceres no. 397/2004 e nº. 0101/06).

\section{Eventos}

O evento observado foi o tempo até a falha do tratamento imunossupressor, definida como o óbito do paciente ou a perda do enxerto. Considerou-se como perda de enxerto o retorno à terapia dialítica por mais de três meses, sem o uso concomitante de imunossupressores. A data do evento foi atribuída pela data do último registro de dispensação de imunossupressores ou pela data do óbito. A censura foi configurada pela perda de seguimento ou fim do acompanhamento, adotando-se a data do último registro referente à imunossupressão. O período de acompanhamento para verificar a ocorrência do evento se estendeu até dezembro de 2004. 


\section{Análise dos gastos}

A análise dos gastos adotou a perspectiva do financiador público e limitou-se aos gastos diretos da atenção à saúde. Para essa análise, foram constituídos dois grupos de participantes, segundo o esquema terapêutico adotado. O primeiro grupo foi composto por indivíduos que utilizaram a ciclosporina no esquema imunossupressor no período de janeiro de 2000 a dezembro de 2004 , mesmo que combinado com outros medicamentos, exceto o tacrolimus. O segundo grupo, por indivíduos que utilizaram o tacrolimus naquele mesmo período, mesmo que combinado com outros medicamentos, exceto a ciclosporina. Foram excluídos da análise pacientes que iniciaram o tratamento usando esquemas com ciclosporina e mudaram para esquemas com tacrolimus, ou vice e versa, durante o período de acompanhamento.

Para o cálculo dos gastos relativos a cada indivíduo, foram considerados todos os procedimentos ambulatoriais e hospitalares registrados durante o período de acompanhamento, ou seja, desde sua entrada até a ocorrência do evento ou censura. A entrada na coorte foi definida pela data de realização do transplante registrada no SIH.

\section{Aferição de gastos}

Para aferir os gastos individuais, foram identificados todos os procedimentos e seus respectivos valores na base AIH/SIH e na base APAC/SIA para cada paciente, considerando o mês como unidade temporal. A partir desses dados, gerouse uma tabela com as informações de valores mensais despendidos (em R\$), por paciente, de acordo com as categorias: hospitalizações, diálises, acompanhamento clínico e exames, medicamentos de alto custo/excepcionais (outros medicamentos, outros agentes imunossupressores e medicamento em estudo). Os valores foram atualizados para dezembro de 2008, com base no Índice de Preços ao Consumidor Amplo (IPCA) do Instituto Brasileiro de Geografia e Estatística (IBGE).

Foram calculados os seguintes indicadores: (i) gasto médio, mediano e total com acompanhamento clínico e exames diagnósticos, hospitalizações, diálises e medicamentos excepcionais no período de acompanhamento a partir do transplante; (ii) gasto médio, mediano e total por paciente, a cada ano de tratamento, a partir do transplante, segundo esquema terapêutico.

\section{Gastos segundo o perfil do paciente}

Para caracterização dos gastos segundo o perfil do paciente, foram selecionadas as seguintes variáveis: sexo, idade, diagnóstico primário da doença renal (glomerulonefrites/nefrite intersticial/pielonefrite, hipertensão/doenças cardiovasculares, diabetes, indeterminada/outras causas), esquema terapêutico (tracrolimus, ciclosporina), tipo de transplante (doador vivo, doador cadavérico), região do centro transplantador e tempo de diálise anterior ao transplante renal. Para essa análise, foram considerados apenas os pacientes que completaram 48 meses de acompanhamento após o transplante.

\section{Análises estatísticas}

Foi realizada análise descritiva para as características estudadas, incluindo distribuições de freqüências, medidas de tendência central e de variabilidade. Diferenças entre valores médios dos gastos segundo o grupo de tratamento e segundo as características dos pacientes foram avaliadas pelo teste $\mathrm{t}$ de Student. O teste Anova foi usado quando a variável não era dicotômica. Adotou-se o nível de significância de 5\%. As análises estatísticas foram realizadas com auxílio do programa “R” versão 2.5.0 (The R Foundation for Statistical Computing, Viena, Áustria; http:// www.r-project.org).

\section{Resultados}

Foram identificados 8.981 pacientes distintos, em 9.298 transplantes renais, com doador vivo ou cadavérico. Do total de pacientes, $89 \%$ apresentam registro de uso de medicamentos no sistema APAC/SIA. Dentre os participantes, o uso exclusivo de esquemas imunossupressores, incluindo a ciclosporina, foi observado em 4.015 pacientes (45\%), e esquemas incluindo o tacrolimus, em 1.159 (13\%), resultando numa coorte de 5.174 indivíduos, no período de janeiro de 2000 a dezembro de 2004.

\section{Estatística descritiva}

Observou-se que a maioria dos pacientes era do sexo masculino (59\%), residia na Região Sudeste $(61 \%)$ e tinha idade mediana de 38 anos no momento do transplante. Os principais diagnósticos primários de insuficiência renal crônica observados foram as glomerulonefrites, nefrites intersticiais, pielonefrites (29\%), hipertensão, doenças cardiovasculares (21\%), diabetes $(5 \%)$ e doenças císticas do rim (2\%), além de outras 
causas e diagnósticos indeterminados. O grande número de pacientes com diagnóstico indeterminado pode estar associado ao quadro clínico avançado da doença renal encontrado no início das terapias de substituição renal, o que dificulta a identificação etiológica. O tipo de transplante mais realizado foi com doador vivo $(62 \%)$, e a maioria dos procedimentos ocorreu nas regiões sudeste e sul do país $(61 \% ; 20 \%)$, em centros transplantadores situados nos municípios de São Paulo, Porto Alegre, Belo Horizonte, Rio de Janeiro e Curitiba (31\%; 7\%; 7\%; 5\%; $4 \%$ ).

\section{Gastos e recursos médicos utilizados}

As Tabelas 1 e 2 apresentam os valores pagos pelo SUS com procedimentos hospitalares, ambulatoriais e medicamentos de alto custo utilizados pelos pacientes durante o período de acompanhamento da coorte. Pode-se observar que as despesas hospitalares de maior impacto foram com os procedimentos de transplante em si, seguidos do procedimento de intercorrência póstransplante e do acompanhamento em âmbito hospitalar dos pacientes que, somados, acumulam $98 \%$ dos gastos hospitalares no período. Já para os recursos ambulatoriais e medicamentos de alto custo consumidos durante o tratamento

Tabela 1

Gasto médio e total com procedimentos hospitalares a partir do transplante renal, durante o período de acompanhamento da coorte. Brasil, $2000-2004$.

\begin{tabular}{|c|c|c|c|c|}
\hline Procedimentos hospitalares & Gasto médio (R\$) & Gasto total (R\$) & $\%$ & $\%$ acumulado \\
\hline Transplante renal receptor - doador vivo & $24.536,72$ & $85.706 .746,59$ & 48,5 & 48,5 \\
\hline Transplante renal receptor - doador cadáver & $32.951,80$ & $69.693 .058,97$ & 39,4 & 88,0 \\
\hline Intercorrência pós-transplante & $2.590,25$ & $13.381 .233,44$ & 7,6 & 95,5 \\
\hline Acompanhamento pós-transplante & $1.304,22$ & $4.131 .755,84$ & 2,3 & 97,9 \\
\hline Transplante de fígado & $77.150,74$ & $231.452,21$ & 0,1 & 98,0 \\
\hline Laparotomia exploradora & $1.128,19$ & $160.203,18$ & 0,1 & 98,1 \\
\hline Insuficiência renal crônica & 666,63 & $159.324,87$ & 0,1 & 98,2 \\
\hline Tratamento cirúrgico fistula & $1.652,29$ & $110.703,66$ & 0,1 & 98,2 \\
\hline Intercorrência - paciente renal crônico (clínica médica) & 773,33 & $109.813,50$ & 0,1 & 98,3 \\
\hline Angioplastia coronariana & $7.666,81$ & $107.335,41$ & 0,1 & 98,4 \\
\hline Septicemia (clínica médica) & $1.964,74$ & $94.307,42$ & 0,1 & 98,4 \\
\hline Nefrectomia total & $1.634,04$ & $73.531,65$ & 0,0 & 98,5 \\
\hline Traqueotomia (inclusive curativos) & $7.135,87$ & $71.358,73$ & 0,0 & 98,5 \\
\hline Pneumonia em adulto & 638,80 & $68.990,92$ & 0,0 & 98,5 \\
\hline Transplante de pâncreas após rim & $22.723,40$ & $68.170,19$ & 0,0 & 98,6 \\
\hline Lombotomia & $1.774,85$ & $62.119,62$ & 0,0 & 98,6 \\
\hline Ureterocistoneostomia & $1.420,26$ & $61.071,28$ & 0,0 & 98,7 \\
\hline Angioplastiade vasos viscerais ou renais com implante & $5.874,50$ & $58.744,98$ & 0,0 & 98,7 \\
\hline Drenagem de abscesso renal ou perirenal & $1.296,90$ & $55.766,60$ & 0,0 & 98,7 \\
\hline Pielonefrite & 391,00 & $52.784,71$ & 0,0 & 98,7 \\
\hline Extração de corpo estranho da bexiga & 746,13 & $51.483,01$ & 0,0 & 98,8 \\
\hline Implante de prótese valvar & $16.706,45$ & $50.119,34$ & 0,0 & 98,8 \\
\hline Tratamento cirúrgico de fístulas ureterais & $1.291,35$ & $49.071,17$ & 0,0 & 98,8 \\
\hline Diabetes sacarino & 544,74 & $43.579,03$ & 0,0 & 98,9 \\
\hline Tratamento clínico AVC isquêmico/hemorrágico agudo & $1.605,54$ & $43.349,58$ & 0,0 & 98,9 \\
\hline Outras afecções do aparelho geniturinário & 353,35 & $42.401,77$ & 0,0 & 98,9 \\
\hline Tratamento cirúrgico de aneurisma periférico arterial/venoso & $1.626,02$ & $40.650,52$ & 0,0 & 98,9 \\
\hline Artroplastia total do quadril não cimentada ou híbrida & $5.449,47$ & $38.146,32$ & 0,0 & 98,9 \\
\hline Outros procedimentos agrupados & ND & $1.857 .935,64$ & 1,1 & 100,0 \\
\hline Total geral & & $176.675 .210,16$ & & \\
\hline
\end{tabular}

ND: não disponível.

Nota: despesas hospitalares para o grupo de pacientes em estudo de janeiro de 2000 a dezembro de 2003 registradas no Sistema de Informações Hospitalares (SIH/SUS); valores atualizados para dezembro de 2008 pelo Índice de Preços ao Consumidor Amplo (IPCA/IBGE). 
Tabela 2

Gasto médio e total com procedimentos ambulatoriais e medicamentos de alto custo a partir do transplante renal, durante o período de acompanhamento da coorte. Brasil, 2000-2004.

\begin{tabular}{|c|c|c|c|c|}
\hline $\begin{array}{l}\text { Medicamentos de alto custo e procedimentos } \\
\text { ambulatoriais }\end{array}$ & Gasto médio (R\$) & Gasto total (R\$) & $\%$ & $\%$ acumulado \\
\hline Ciclosporina & 266,59 & $48.627 .302,94$ & 31,9 & 31,9 \\
\hline Micofenolato mofetil & 680,38 & $42.368 .051,99$ & 27,1 & 59,1 \\
\hline Tacrolimus & 896,97 & $27.942 .465,22$ & 17,9 & 76,9 \\
\hline Consulta para acompanhamento de transplante & 147,76 & $15.092 .616,86$ & 9,9 & 86,9 \\
\hline Hemodiálise & $1.293,25$ & $5.068 .257,37$ & 3,2 & 90,0 \\
\hline Dosagem de ciclosporina & 110,91 & $3.298 .747,58$ & 2,2 & 92,2 \\
\hline Sirolimus & 704,87 & $2.715 .881,38$ & 1,7 & 94,0 \\
\hline Diálise peritonial & $1.481,31$ & $1.755 .359,14$ & 1,1 & 95,1 \\
\hline Outros medicamentos de alto custo & $3.866,02$ & $1.596 .667,12$ & 1,1 & 96,2 \\
\hline Azatioprina & 21,92 & $1.349 .768,15$ & 0,9 & 97,1 \\
\hline Eritropoetina & 243,08 & $1.329 .167,10$ & 0,9 & 97,9 \\
\hline Dosagem de tacrolimus & 105,06 & $1.248 .700,70$ & 0,8 & 98,7 \\
\hline Exames - análises clínicas & 12,29 & $551.508,53$ & 0,4 & 99,1 \\
\hline Medicamentos reguladores do cálcio & 62,71 & $236.568,06$ & 0,2 & 99,3 \\
\hline Cintilografia & 221,62 & $130.532,67$ & 0,1 & 99,3 \\
\hline Radiodiagnóstico & 50,63 & $112.045,53$ & 0,1 & 99,4 \\
\hline Dosagem de cd4/cd3 em transplantado & 205,00 & $107.214,75$ & 0,1 & 99,5 \\
\hline Hidróxido de ferro & 35,22 & $94.426,05$ & 0,1 & 99,6 \\
\hline Acesso para diálise & 100,19 & $93.786,29$ & 0,1 & 99,6 \\
\hline Medicamentos antivirais & 78,32 & $81.999,85$ & 0,1 & 99,7 \\
\hline Biópsia & 329,96 & $80.839,51$ & 0,1 & 99,7 \\
\hline Cirurgia ambulatorial em aparelho visual & $1.032,64$ & $75.382,45$ & 0,0 & 99,8 \\
\hline Quimioterapia & 690,59 & $75.274,16$ & 0,0 & 99,8 \\
\hline Ressonância magnética & 367,02 & $45.510,88$ & 0,0 & 99,8 \\
\hline Tomografia & 148,28 & $44.631,38$ & 0,0 & 99,9 \\
\hline Ecocrafia em paciente transplantado & 21,70 & $43.243,69$ & 0,0 & 99,9 \\
\hline Medicamentos quelantes & 344,17 & $40.955,37$ & 0,0 & 99,9 \\
\hline Estatinas e fibratos & 54,54 & $36.382,96$ & 0,0 & 99,9 \\
\hline Outros procedimentos agrupados & ND & $78.074,94$ & 0,1 & 100,0 \\
\hline Total geral & 249,45 & $154.321 .362,61$ & 100,0 & \\
\hline
\end{tabular}

ND: não disponível.

Nota: despesas ambulatoriais e medicamentos de alto custo para o grupo de pacientes em estudo de janeiro de 2000 a dezembro de 2004 registradas no Sistema de Informações Ambulatoriais (APAC/SIA/SUS); valores atualizados para dezembro de 2008 pelo Índice de Preços ao Consumidor Amplo (IPCA/IBGE).

observou-se, como esperado, relevante impacto na despesa referente ao uso dos medicamentos imunossupressores, representando cerca de $80 \%$ dos gastos no sistema APAC/SIA. A utilização de procedimentos relacionados à hemodiálise ou às diálises peritoneais representou em conjunto uma despesa de $4 \%$ no grupo em estudo.

\section{Evolução dos gastos e recursos médicos utilizados por grupo de tratamento}

As Figuras 1 e 2 apresentam a evolução do gasto mediano anual para a manutenção do transplante renal. Pode-se observar que os pacientes que receberam esquema imunossupressor com tacrolimus apresentaram maior gasto mediano ao longo do acompanhamento, quando comparados ao grupo da ciclosporina. O mesmo pode ser observado naqueles pacientes que realizaram transplante com doador cadáver.

Os gastos totais e gastos por paciente com o uso de esquemas terapêuticos, seja com tacrolimus, seja com a ciclosporina, além de outros medicamentos imunossupressores, estão apresentados em maior detalhe na Tabela 3. Observaram-se, na evolução do gasto mediano global por paciente, despesas significativamente inferiores no grupo da ciclosporina, ao longo do período de 
Figura 1

Evolução do gasto mediano total para manutenção do transplante renal no Sistema Único de Saúde (SUS), por paciente, a cada ano de tratamento, segundo esquema terapêutico. Brasil, 2000-2004.

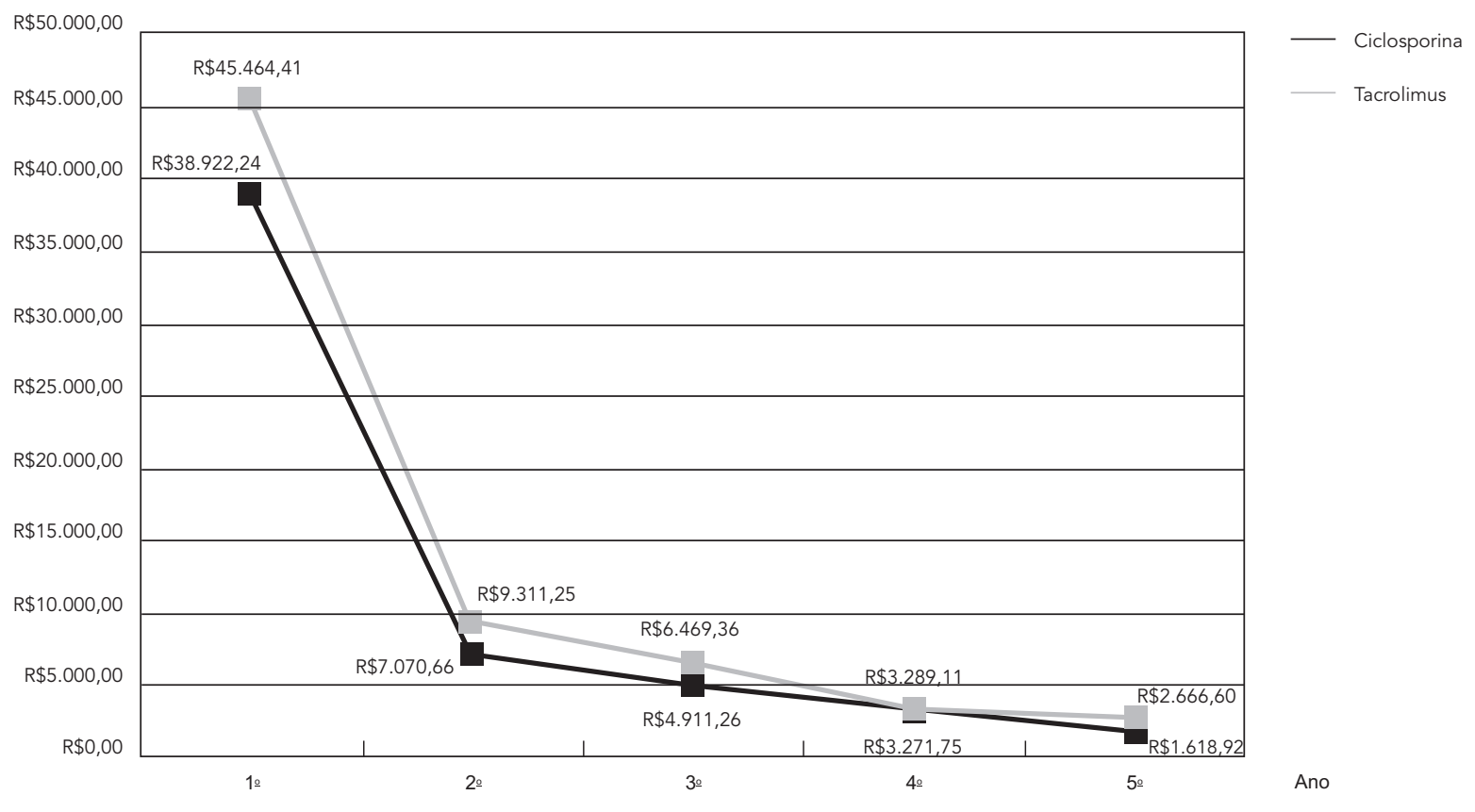

acompanhamento, em comparação com o grupo do tacrolimus. Quando se analisam os gastos totais para o conjunto de pacientes, em cada esquema terapêutico, verificou-se que, no primeiro ano, o uso de recursos hospitalares representou a maior parcela desses gastos em ambos os grupos, tendo em vista o alto custo dos procedimentos de transplante. Assim, os gastos hospitalares atingiram $75 \%$ do total gasto no grupo da ciclosporina e $65 \%$ no do tacrolimus. Nos anos posteriores, observou-se uma participação menor das despesas hospitalares no gasto total, situando-se entre $1 \%$ a $6 \%$. Além disso, constatou-se a utilização de outros recursos terapêuticos relevantes, com destaque para o peso do acompanhamento clínico ambulatorial e dos exames diagnósticos que alcançaram 14\%, no grupo da ciclosporina, e 9\%, no grupo do tacrolimus, no quarto ano de tratamento. A utilização concomitante de outros medicamentos imunossupressores fornecidos pelo programa também apresenta forte impacto no gasto total ao longo do período observado.

\section{Gastos segundo o perfil dos pacientes}

A Tabela 4 apresenta as despesas do SUS referentes aos gastos medianos hospitalares, ambulatoriais e com medicamentos de alto custo, para os 2.176 pacientes que completaram 48 meses de acompanhamento após o transplante. Ao se estratificarem esses gastos, pelas variáveis sócio-demográficas e clínicas, observou-se despesa significativamente superior em pacientes cujo centro transplantador era localizado na Região Nordeste (R\$67.251,74), ao passo que os transplantados na Região Norte apresentaram menor gasto $(\mathrm{R} \$ 58.542,82)$. Também se verificou maior gasto mediano no período estudado para os pacientes que receberam enxerto de doador cadáver $(\mathrm{R} \$ 73.029,58)$ e para aqueles que realizaram diálises por mais de 24 meses antes do transplante $(\mathrm{R} \$ 66.645,83)$. Com relação à terapêutica medicamentosa, pode-se constatar que os pacientes submetidos ao esquema imunossupressor que incluía tacrolimus apresentaram, no período, gasto total $(\mathrm{R} \$ 77.684,96)$ superior ao dos pacientes que foram tratados com esquemas envolvendo a ciclosporina $(\mathrm{R} \$ 61.278,32)$. As 
Evolução do gasto mediano total para manutenção do transplante renal no SUS, por paciente, a cada ano de tratamento, segundo tipo de doador. Brasil, 2000-2004.

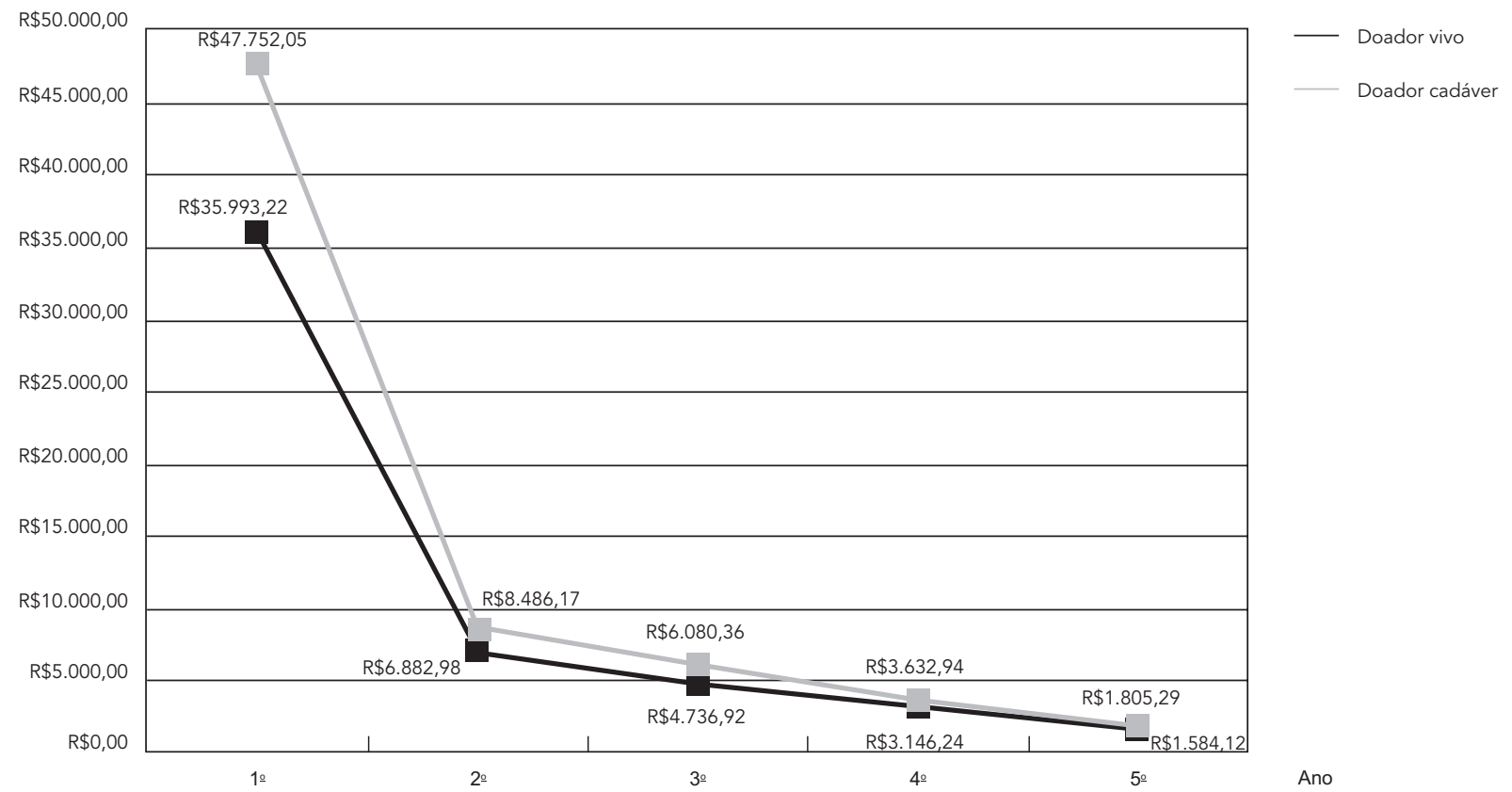

variáveis remanescentes constantes da Tabela 4 não apresentaram significância estatística.

\section{Discussão}

Em relação às características da coorte estudada, verificou-se que a maioria dos pacientes era do sexo masculino, residia na Região Sudeste do país e apresentava como diagnóstico primário mais freqüente de insuficiência renal crônica as glomerulonefrites/nefrites intersticiais/pielonefrites, a hipertensão/doenças cardiovasculares e as causas indeterminadas. Esta última condição é condizente com a realidade brasileira no que diz respeito ao alto grau de complexidade da insuficiência renal crônica, geralmente apresentada pelos pacientes no início das terapias de substituição renal, dificultando o diagnóstico da doença de base.

Os gastos medianos totais, nos 48 meses após o transplante, variaram significativamente segundo a região do hospital transplantador. Observou-se despesa superior em pacientes transplantados na Região Nordeste, enquanto os da Região Norte apresentaram menor gasto. Esse fato pode ser explicado, pelo menos em parte, pelas dificuldades de acesso aos serviços de saúde especializados na Região Norte.

Diversos fatores podem influenciar a sobrevida do enxerto a curto e longo prazo, como o grau de compatibilidade dos antígenos leucocitários humanos, as crises de rejeição aguda, necrose tubular aguda no pós-operatório, imunossupressão inadequada, tempo de isquemia do órgão doado e incompatibilidade no tamanho deste para as necessidades do receptor, raça, sexo, idade do doador e do receptor, hiperlipidemia, infecções, hipertensão arterial, tempo em diálise, dentre outros 10,11,12,13. O presente estudo não dispunha de informações para a maioria desses fatores, mas foi possível evidenciar maior gasto mediano no período estudado para os pacientes que receberam enxerto de doador cadáver e para aqueles que realizaram diálises por mais de 24 meses antes do transplante. Em ambas as situações, observam-se maiores probabilidades de complicações futuras, seja devido ao tempo de isquemia fria em órgãos de origem cadavérica em um país de dimensões continentais como o 
Tabela 3

Gastos relativos à utilização de recursos hospitalares, ambulatoriais e medicamentos de alto custo por pacientes, a partir do transplante renal, segundo grupo de tratamento, durante o período de acompanhamento da coorte. Brasil, 2000-2004.

\begin{tabular}{|c|c|c|c|c|c|c|c|c|c|}
\hline \multirow{2}{*}{$\begin{array}{l}\text { Categoria do recurso } \\
\text { utilizado }\end{array}$} & \multicolumn{4}{|c|}{ Ciclosporina } & \multicolumn{4}{|c|}{ Tacrolimus } & \multirow[t]{2}{*}{ Valor de $p$ * } \\
\hline & Pacientes & $\begin{array}{c}\text { Gasto } \\
\text { mediano (R\$) }\end{array}$ & $\begin{array}{c}\text { Gasto } \\
\text { total (R\$) }\end{array}$ & $\%$ & Pacientes & $\begin{array}{c}\text { Gasto } \\
\text { mediano (R\$) }\end{array}$ & $\begin{array}{c}\text { Gasto } \\
\text { total (R\$) }\end{array}$ & $\%$ & \\
\hline 1 으 ano de tratamento & & \multicolumn{2}{|c|}{$n=4.015$} & \multicolumn{5}{|c|}{$n=1.159$} & \\
\hline $\begin{array}{l}\text { Medicamento } \\
\text { em estudo }\end{array}$ & 4.015 & $4.955,65$ & $23.284 .570,43$ & 14,0 & 1.159 & $9.761,85$ & $13.528 .509,56$ & 24,1 & 0,00 \\
\hline $\begin{array}{l}\text { Outros } \\
\text { imunossupressores }\end{array}$ & 3.760 & 531,90 & $10.525 .242,33$ & 6,3 & 1.133 & $2.313,90$ & $3.506 .695,48$ & 6,2 & 0,01 \\
\hline Outros medicamentos & 897 & 371,76 & $610.019,27$ & 0,4 & 267 & 273,66 & $160.124,90$ & 0,3 & 0,35 \\
\hline $\begin{array}{l}\text { Acompanhamento } \\
\text { clínico e exames }\end{array}$ & 3.127 & $1.925,78$ & $6.098 .910,37$ & 3,7 & 1.073 & $2.263,44$ & $2.366 .893,84$ & 4,2 & 0,00 \\
\hline Diálises & 517 & 675,44 & $1.483 .954,15$ & 0,9 & 137 & 806,83 & $451.592,01$ & 0,8 & 0,44 \\
\hline Hospitalizações & 4.015 & $27.516,36$ & $124.518 .500,67$ & 74,8 & 1.159 & $27.600,40$ & $36.217 .593,54$ & 64,4 & 0,54 \\
\hline Total (AIH e APAC) ** & 4.015 & $38.922,24$ & $166.460 .046,37$ & 100,0 & 1.159 & $45.464,41$ & $56.214 .144,55$ & 100,0 & 0,00 \\
\hline 2o ano de tratamento & & \multicolumn{2}{|c|}{$\mathrm{n}=3.902$} & \multicolumn{5}{|c|}{$n=1.148$} & \\
\hline $\begin{array}{l}\text { Medicamento } \\
\text { em estudo }\end{array}$ & 3.902 & $2.873,04$ & $13.815 .413,20$ & 42,8 & 1.148 & $5.206,24$ & $8.012 .461,28$ & 60,9 & 0,00 \\
\hline $\begin{array}{l}\text { Outros } \\
\text { imunossupressores }\end{array}$ & 3.698 & $1.065,35$ & $10.677 .202,29$ & 33,1 & 1.099 & $1.241,39$ & $2.770 .069,70$ & 21,0 & 0,00 \\
\hline Outros medicamentos & 243 & 230,09 & $172.249,60$ & 0,5 & 100 & 269,72 & $62.796,14$ & 0,5 & 0,65 \\
\hline $\begin{array}{l}\text { Acompanhamento } \\
\text { clínico e exames }\end{array}$ & 3.337 & $1.207,24$ & $4.519 .033,36$ & 14,0 & 1.042 & $1.145,33$ & $1.283 .278,57$ & 9,7 & 0,00 \\
\hline Diálises & 143 & $2.433,94$ & $1.052 .104,82$ & 3,3 & 46 & $3.053,98$ & $283.580,58$ & 2,2 & 0,45 \\
\hline Hospitalizações & 822 & $1.192,61$ & $2.032 .714,49$ & 6,3 & 198 & $1.522,56$ & $749.937,21$ & 5,7 & 0,00 \\
\hline Total (AlH e APAC) ** & 3.902 & $7.070,66$ & $32.292 .064,29$ & 99,9 & 1.148 & $9.311,25$ & $13.164 .281,60$ & 100,0 & 0,00 \\
\hline 3ㅇ ano de tratamento & & \multicolumn{2}{|c|}{$\mathrm{n}=2.876$} & \multicolumn{5}{|c|}{$n=708$} & \\
\hline $\begin{array}{l}\text { Medicamento } \\
\text { em estudo }\end{array}$ & 2.876 & $2.029,16$ & $6.287 .778,20$ & 35,3 & 708 & $3.642,60$ & $3.478 .947,20$ & 60,1 & 0,00 \\
\hline $\begin{array}{l}\text { Outros } \\
\text { imunossupressores }\end{array}$ & 2.734 & $1.053,62$ & $7.192 .933,26$ & 40,4 & 661 & 809,42 & $1.201 .858,84$ & 20,8 & 0,00 \\
\hline Outros medicamentos & 198 & 202,00 & $93.170,23$ & 0,5 & 62 & 182,34 & $27.981,76$ & 0,5 & 0,90 \\
\hline $\begin{array}{l}\text { Acompanhamento } \\
\text { clínico e exames }\end{array}$ & 2.333 & 983,98 & $2.569 .594,48$ & 14,4 & 600 & 852,44 & $574.884,07$ & 9,9 & 0,00 \\
\hline Diálises & 97 & $2.712,12$ & $575.588,22$ & 3,2 & 36 & $2.153,80$ & $218.884,24$ & 3,8 & 0,92 \\
\hline Hospitalizações & 439 & $1.210,15$ & $1.061 .771,89$ & 6,0 & 75 & $1.769,27$ & $280.390,54$ & 4,8 & 0,01 \\
\hline Total (AlH e APAC) ** & 2.876 & $4.911,26$ & $17.805 .359,68$ & 99,9 & 708 & $6.469,36$ & $5.791 .310,72$ & 99,9 & 0,00 \\
\hline 4 o ano de tratamento & & \multicolumn{2}{|c|}{$\mathrm{n}=1.847$} & \multicolumn{5}{|c|}{$n=329$} & \\
\hline $\begin{array}{l}\text { Medicamento } \\
\text { em estudo }\end{array}$ & 1.847 & $1.292,00$ & $2.692 .916,57$ & 33,9 & 329 & $1.972,04$ & $985.412,21$ & 51,4 & 0,00 \\
\hline $\begin{array}{l}\text { Outros } \\
\text { imunossupressores }\end{array}$ & 1.758 & 694,60 & $3.452 .020,60$ & 43,5 & 302 & 244,00 & $333.105,11$ & 17,4 & 0,00 \\
\hline Outros medicamentos & 144 & 156,99 & $44.532,97$ & 0,6 & 28 & 158,11 & $292.370,34$ & 15,2 & 0,02 \\
\hline $\begin{array}{l}\text { Acompanhamento } \\
\text { Clínico e Exames }\end{array}$ & 1.423 & 635,20 & $1.098 .575,38$ & 13,8 & 270 & 460,47 & $164.339,45$ & 8,6 & 0,00 \\
\hline Diálises & 47 & $3.757,30$ & $282.237,76$ & 3,6 & 9 & $7.471,55$ & $113.157,78$ & 5,9 & 0,02 \\
\hline Hospitalizações & 146 & 958,45 & $353.708,97$ & 4,5 & 11 & $1.440,38$ & $14.915,53$ & 0,8 & 0,33 \\
\hline Total (AlH e APAC) ** & 1.847 & $3.271,75$ & $7.936 .531,89$ & 99,8 & 329 & $3.289,11$ & $1.917 .507,64$ & 99,3 & 0,00 \\
\hline
\end{tabular}

AlH: Autorizações de Internação Hospitalar; APAC: Autorização de Procedimentos Ambulatoriais de Alta Complexidade.

* Valor de p calculado pelo teste t para avaliar diferenças entre os valores médios dos grupos.

** O total AlH e APAC representam o gasto mediano total e não a soma dos valores da coluna.

Nota: despesas ambulatoriais e medicamentos de alto custo registrados no Sistema de Informações Hospitalares (SIH/SUS) e ambulatorial (APAC/SIA), para o grupo de pacientes em estudo de janeiro de 2000 a dezembro de 2004; valores atualizados para dezembro de 2008 pelo Índice de Preços ao Consumidor Amplo (IPCA/IBGE). 
Gasto mediano total com internações hospitalares, ambulatoriais e medicamentos de alto custo nos 48 meses a partir do transplante, distribuídos de acordo com variáveis demográficas e clínicas na coorte. Brasil, 2000-2004.

\begin{tabular}{|c|c|c|c|}
\hline \multirow[t]{2}{*}{ Variável } & \multicolumn{2}{|c|}{ Coorte (48 meses) } & \multirow[t]{2}{*}{ Valor de $p$ * } \\
\hline & $n=2.176$ & $\begin{array}{l}\text { Gasto mediano } \\
\text { total (R\$) }\end{array}$ & \\
\hline 1) Sexo & & & 0,53 \\
\hline Masculino & 1.269 & $63.797,21$ & \\
\hline Feminino & 907 & $62.732,08$ & \\
\hline 2) Idade no transplante (anos) & & & 0,35 \\
\hline$>38$ & 1.075 & $63.776,15$ & \\
\hline$\leq 38$ & 1.101 & $62.875,61$ & \\
\hline $\begin{array}{l}\text { 3) Causa de insuficiência renal } \\
\text { crônica - diagnóstico primário }\end{array}$ & & & 0,75 \\
\hline Nefrites: glomerulo/intersticial/pielo & 697 & $63.738,88$ & \\
\hline $\begin{array}{l}\text { Hipertensão/Doenças } \\
\text { cardiovasculares }\end{array}$ & 473 & $64.432,44$ & \\
\hline Diabetes & 95 & $59.918,61$ & \\
\hline Indeterminada/Outras causas & 911 & $62.541,85$ & \\
\hline 4) Região do hospital transplantador & & & $<0,01$ \\
\hline Sudeste & 1.364 & $63.587,61$ & \\
\hline Sul & 413 & $60.882,65$ & \\
\hline Nordeste & 203 & $67.251,74$ & \\
\hline Centro-Oeste & 152 & $65.224,00$ & \\
\hline Norte & 44 & $58.542,82$ & \\
\hline 5) Tipo de transplante & & & $<0,01$ \\
\hline Doador cadáver & 800 & $73.029,58$ & \\
\hline Doador vivo & 1.376 & $58.076,53$ & \\
\hline $\begin{array}{l}\text { 6) Tempo mediano de diálise antes } \\
\text { do transplante (meses) }\end{array}$ & & & $<0,01$ \\
\hline$>24$ & 991 & $66.645,83$ & \\
\hline$\leq 24$ & 1.185 & $59.771,09$ & \\
\hline 7) Esquema terapêutico & & & $<0,01$ \\
\hline Tacrolimus & 329 & $77.684,96$ & \\
\hline Ciclosporina & 1.847 & $61.278,32$ & \\
\hline
\end{tabular}

* Valor de p calculado pelo teste t para avaliar diferenças entre os valores médios dos grupos. O teste Anova foi usado quando a variável não era dicotômica.

Nota: valores atualizados para dezembro de 2008 pelo Índice de Preços ao Consumidor Amplo (IPCA/IBGE).

Brasil, seja pela necessidade cotidiana de procedimentos dialíticos por longo tempo.

As taxas de sobrevida em pacientes transplantados, após um ano do enxerto, são ligeiramente maiores para aqueles que receberam o órgão de doador relacionado, do que para os que receberam o órgão de doador cadáver. Isso acontece porque vários fatores interferem no sucesso do procedimento, ou seja, é necessário que tanto o doador quanto o paciente preencham critérios específicos para doação e recepção do órgão a ser transplantado. Resultados menos favoráveis em pacientes que recebem o órgão de doador cadáver persistem a longo prazo 6,12.
No SUS, são realizados transplantes renais com doadores vivos e cadáveres, e quase $80 \%$ dos transplantes com doadores vivos são realizados com órgãos doados de parentes próximos 6 . Sabe-se que o transplante intervivos apresenta menos episódios de rejeição crônica e aguda que os realizados com doador cadáver, o que ajuda a explicar o menor gasto mediano nesse estrato de pacientes em 48 meses de acompanhamento.

Com relação à terapêutica medicamentosa, pode-se constatar que os pacientes submetidos ao esquema imunossupressor que incluía tacrolimus apresentaram maior gasto mediano total 
ao longo do acompanhamento, quando comparados ao grupo da ciclosporina. O tacrolimus é apresentado como inovação terapêutica superior à ciclosporina, sendo, por isso, mais caro, o que reflete diretamente na despesa do SUS com os pacientes em uso desses medicamentos; observaram-se também maiores gastos hospitalares por paciente no grupo do tacrolimus que no grupo da ciclosporina. Como a utilização concomitante de outros medicamentos imunossupressores é recomendada e fornecida pelo Programa de Medicamentos Excepcionais, observa-se, no grupo da ciclosporina, um crescimento na despesa com esses produtos, enquanto no grupo do tacrolimus sucede o contrário. Esse fato pode estar apoiado na confiança que o prescritor deposita na inovação terapêutica, dispensando o uso concomitante dos outros agentes recomendados pelo protocolo clínico do Ministério da Saúde, o que, por sua vez, pode ter reflexos na manutenção do transplante, resultando em sessões adicionais de diálises e hospitalizações.

Mesmo com a maior disponibilidade de drogas imunossupressoras, o maior benefício desses medicamentos parece se concentrar na redução do número de episódios de rejeição aguda ${ }^{13}$, que é um dos principais fatores de risco para a nefropatia crônica do enxerto. Entretanto, seus efeitos na sobrevida a curto e longo prazo têm sido menos expressivos, apesar da adição de novos fármacos, a exemplo do tacrolimus, ao esquema terapêutico inicial com azatioprina e prednisona. A literatura não tem confirmado uniformemente vantagens da imunossupressão ancorada no tacrolimus 12. Esse medicamento estaria mais recomendado para o transplante hepático do que o renal, e um dos seus efeitos adversos mais descritos é a ocorrência de diabetes 14,15,16,17.

No Brasil, o esquema terapêutico de manutenção mais freqüente inclui a ciclosporina, mas observa-se crescente incremento do número de pacientes que já iniciam o tratamento com tacrolimus. Esta situação, de acordo com os protocolos clínicos adotados pelo país, não deveria ocorrer com frequência. Preconiza-se iniciar a imunossupressão com ciclosporina e, após avaliação clínica, alternativamente, utilizar tacrolimus, recomendação que parece não estar sendo seguida. Do ponto de vista fármaco-econômico, dados de comercialização obtidos do Banco Nacional de Preços do Ministério da Saúde (BNP; http://bpreco.saude.gov.br) registram um valor por tratamento mensal com tacrolimus cerca de três vezes maior do que com a ciclosporina. Ambos os medicamentos são majoritariamente adquiridos pelo SUS, contudo, para conhecer o impacto econômico e a relação de custo-efetividade entre as duas alternativas imunossupressoras, é preciso levar em conta, além dos recursos econômicos consumidos 10,18, intercorrências clínicas, reações adversas e efeitos colaterais da terapêutica, principalmente a sobrevida do paciente com enxerto funcional e sua respectiva qualidade de vida. Dessa forma, seria possível estabelecer qual droga tem se mostrado mais vantajosa na perspectiva do SUS e dos pacientes que as utilizam, desafio que deverá ser objeto de investigações futuras.

\section{Considerações finais}

No Brasil, o SUS tem sido majoritariamente responsável pela realização e manutenção do transplante renal, fornecendo medicamentos de alto custo, diálises, acompanhamento clínico, exames diagnósticos, bem como hospitalizações necessárias, a despeito das dificuldades crônicas de financiamento vivenciadas pelo sistema. Ao final de 48 meses de acompanhamento dos pacientes que permaneceram no estudo, a compilação das despesas na perspectiva do SUS, adotada neste estudo, permitiu constatar que o gasto mediano total com procedimentos destinados à manutenção do transplante renal, em pacientes que utilizaram esquemas terapêuticos com tracrolimus, foi superior ao daqueles em esquemas com ciclosporina. 


\section{Resumo}

No Brasil, o Sistema Único de Saúde (SUS) é responsável maioria dos transplantes renais. Para a manutenção dessas intervenções, os protocolos recomendam uso da ciclosporina ou tacrolimus, associado com corticosteróides e azatioprina ou micofenolato. Na perspectiva do SUS, realizou-se análise econômica sobre recursos ambulatoriais, hospitalares e medicamentos utilizados por paciente e grupo terapêutico. Foi construída coorte de 2000 a 2004, com 5.174 pacientes em transplantes renais e em uso de ciclosporina ou tacrolimus, identificados por relacionamento probabilístico em registros do SUS. A coorte continha 4.015 pacientes em uso de ciclosporina e 1.159 com tacrolimus. A maioria era do sexo masculino, idade $\leq 38$ anos, cujos diagnósticos primários mais freqüentes eram nefrites, doenças cardiovasculares e causas indeterminadas. Após 48 meses, observou-se gasto superior para transplantes renais em hospitais do Nordeste, doador cadáver, naqueles em diálises $>24$ meses antes do transplantes renais e no grupo do tacrolimus. Constatou-se maior gasto total com recursos hospitalares, ambulatoriais e medicamentos para os transplantes renais em pacientes com esquemas com tacrolimus, quando comparados com o grupo da ciclosporina.

Transplante Renal; Gastos em Saúde; Economia da Saúde

\section{Colaboradores}

A. A. Guerra Junior, F. A. Acúrcio, E. I. G. Andrade e M. L. Cherchiglia contribuíram com o desenho do estudo, análise e interpretação de dados, elaboração e revisão crítica do texto e aprovação da versão final do artigo. C. C. Cesar, O. V. Queiroz e G. D. Silva contribuíram com a análise e interpretação de dados, elaboração e revisão crítica do texto e aprovação da versão final do artigo.

\section{Agradecimentos}

A pesquisa teve o apoio financeiro do Conselho Nacional de Desenvolvimento Científico e Tecnológico (CNPq), Fundação de Amparo à Pesquisa do Estado de Minas Gerais (FAPEMIG) e Ministério da Saúde, além do apoio logístico do Departamento de Medicina Preventiva e Social, do Departamento de Farmácia Social e do Núcleo de Educação em Saúde Coletiva da Universidade Federal de Minas Gerais.

\section{Referências}

1. Secretaria de Atenção à Saúde, Ministério da Saúde. Política nacional ao portador de doença renal. Textos básicos em saúde. Brasília: Ministério da Saúde; 2004.

2. Sociedade Brasileira de Nefrologia. Perfil da doença renal crônica. O desafio brasileiro. http://www. sbn.org.br/noticias/DossieFinal.pdf (acessado em 13/Jun/08).

3. Machnicki G, Louiza S, Schnitzler MA. Economics of transplantation: a review of the literature. Transplant Rev 2006; 20:61-75.

4. Ministério da Saúde. Portaria SAS/MS 221, de lo de abril de 2002. Aprova o protocolo clínico e diretrizes terapêuticas de medicamentos excepcionais e de alto custo no SUS. Diário Oficial da União 2002; 2 abr.
5. Woodroffe R, Yao GL, Meads C, Bayliss S, Ready A, Raftery J, et al. Clinical and cost-effectiveness of newer immunosuppressive regimens in renal transplantation: a systematic review and modeling study. Health Technol Assess 2005; 9(21).

6. Sociedade Brasileira de Nefrologia. Diretrizes em transplante renal. http://www.sbn.org.br/Diretri zes/tx.htm (acessado em 13/Jun/08).

7. Coeli CM, Camargo Jr. KR. Avaliação de diferentes estratégias de blocagem no relacionamento probabilístico de registros. Rev Bras Epidemiol 2002; 5:185-95. 
8. Cherchiglia ML, Guerra Júnior AA, Andrade EIG, Machado CJ, Acúrcio FA, Meira Júnior W, et al. A construção da base de dados nacional em Terapia Renal Substitutiva (TRS) centrada no indivíduo: aplicação do método de linkage determinístico probabilístico. Rev Bras Estud Popul 2007; 24:163-7.

9. Queiroz OV, Guerra Júnior AA, Machado CJ, Andrade EIG, Meira Júnior W, Acúrcio FA, et al. A construção da Base Nacional de Dados em Terapia Renal Substitutiva (TRS) centrada no indivíduo: relacionamento dos registros de óbitos pelo subsistema de Autorização de Procedimentos de Alta Complexidade (APAC/SIA/SUS) e pelo Sistema de Informações sobre Mortalidade (SIM) - Brasil, 2000-2004. Epidemiol Serv Saúde 2009; 18:107-20.

10. Gentil MA, Cantarell AC, Roncero FMG, Franco JEM, López MMM. Impact of the new drugs in the cost of maintenance immunosuppression of renal transplantation. Is it justified? Nephrol Dial Transplant 2004; 19:77-82.

11. Abbud Filho M, Ramalho HJ. Revisão/atualização em transplante renal: novos agentes imunossupressores. J Bras Nefrol 1997; 19:215-23.

12. Hwang AH, Cho YW, Cicciarelli J, Mentser M, IwakiY, Hardy B. Risk factors for short- and long-term survival of primary cadaveric renal allografts in pediatric recipients: a UNOS analysis. Transplantation 2005 ; 80:466-70.
13. Bunnapradist S, Daswani A, Takemoto SK. Graft survival following living-donor renal transplantation: a comparison of tacrolimus and cyclosporine microemulsion with mycophenolate mofetil and steroids. Transplantation 2003; 76:10-5.

14. Bastos Júnior MAV, Oliveira MMS, Castro SH, Cunha EF, Moraes ERS, Ruzzani F, et al. Fatores de risco para o desenvolvimento de diabetes mellitus pós-transplante renal. Arq Bras Endocrinol Metab 2005; 49:271-7.

15. Mayer AD, Dmitrewski J, Squifflet J-P, Besse T, Grabensee B, Klein B, et al. Multicenter randomized trial comparing tacrolimus (FK506) and cyclosporine in the prevention of renal allograft rejection: a report of the European Tacrolimus Multicenter Renal Study Group. Transplantation 1997; 64:436-43.

16. Margreiter R. Efficacy and safety of tacrolimus compared with ciclosporin microemulsion in renal transplantation: a randomised multicentre study. Lancet 2002; 359:741-6.

17. Kramer BK, Montagnino G, Del Castillo D, Margreiter R, Sperschneider H, Olbricht CJ, et al. Efficacy and safety of tacrolimus compared with cyclosporin A microemulsion in renal transplantation: 2 year follow-up results. Nephrol Dial Transplant 2005; 20:968-73.

18. Orme ME, Jurewicz WA, Kumar N, McKechnie TL The cost effectiveness of tacrolimus versus microemulsified cyclosporin: a 10-year model of renal transplantation outcomes. Pharmacoeconomics 2003; 21:1263-76.

Recebido em 20/Mar/2009

Versão final reapresentada em 09/Set/2009

Aprovado em 16/Set/2009 